\title{
Impact of primary tumor location as a predictive factor in patients suffering from colorectal cancer treated with cytotoxic anticancer agents based on the collagen gel droplet-embedded drug sensitivity test
}

\author{
TAKUMI OCHIAI ${ }^{1}$, KAZUHIKO NISHIMURA $^{1}$, TOMOO WATANABE ${ }^{1}$, MASAYUKI KITAJIMA $^{1}$, \\ AKINORI NAKATANI ${ }^{1}$, KIICHI NAGAYASU ${ }^{1}$, NAOKI SAKUYAMA ${ }^{1}$, TSUYOSHI SATO ${ }^{1}$, \\ KENJI KISHINE $^{1}$, YU ABE ${ }^{1}$ and ISAO NAGAOKA ${ }^{2}$ \\ ${ }^{1}$ Department of Surgery, Tobu Chiiki Hospital, Tokyo Metropolitan Health and Medical Treatment Corporation, \\ Tokyo 125-8512; ${ }^{2}$ Department of Host Defense and Biochemical Research, \\ Juntendo University School of Medicine, Tokyo 113-8421, Japan
}

Received June 9, 2018; Accepted November 20, 2018

DOI: $10.3892 / \mathrm{ol} .2018 .9805$

\begin{abstract}
In recent studies, better clinical outcomes for patients with left-sided colon cancer (CC) compared with right-sided $\mathrm{CC}$ have been reported; however, in such investigations, the chemotherapy regimens included molecular-targeting agents. To the best of our knowledge, the impact of primary tumor location as a predictive factor in patients suffering from CC treated with cytotoxic anticancer agents alone has not been investigated. The aim of the present study was to determine the impact of the primary tumor location as a predictive factor of patients undergoing the following cytotoxic anticancer agent regimens: Leucovorin and fluorouracil + oxaliplatin (FOLFOX) or Leucovorin and fluorouracil + irinotecan (FOLFIRI), using the collagen gel droplet-embedded drug sensitivity test (CD-DST). Between March 2008 and April 2017, tumor specimens were obtained from 133 patients suffering from colorectal cancer (CRC) who had not received preoperative chemotherapy. CD-DST was performed and the growth inhibition rate (IR) was determined in FOLFOX and FOLFIRI regimens. The associations between tumor location and IR values for each condition were evaluated. In the present study, the prognosis of patients receiving palliative chemotherapy as well as treatment with molecularly-targeted agents was also investigated. There were no significant differences in the IRs (\%) of the two regimens using CD-DST for right-sided tumors compared with left-sided tumors, including
\end{abstract}

Correspondence to: Dr Takumi Ochiai, Department of Surgery, Tobu Chiiki Hospital, Tokyo Metropolitan Health and Medical Treatment Corporation, 5-14-1 Kameari, Tokyo 125-8512, Japan E-mail: takumi_ochiai@tokyo-hmt.jp

Key words: colorectal cancer, tumor location, collagen gel droplet-embedded drug sensitivity test, predictive factor, cytotoxic anticancer agent or excluding the rectum. The median survival times of patients with right $\mathrm{CC}$ and left $\mathrm{CC}$ who had received palliative chemotherapy and treatment with molecularly-targeted agents were 960 and 1,348 days, respectively. Primary tumor location did not represent a predictive factor for the efficacy of treatment with cytotoxic anticancer agent regimens using CD-DST. However, patients suffering from left-sided CC were revealed to exhibit better clinical outcomes compared with patients suffering from right-sided CC when molecularly-targeted agent regimens were administered. Therefore, the results of the present study suggested that molecularly-targeted agents rather than cytotoxic anticancer agents may result in improved clinical outcomes for patients with CRC suffering from left-sided tumors.

\section{Introduction}

Colorectal cancer (CRC) is one of the most common cancers types worldwide $(1,2)$. Within the last ten years in particular, the prognosis for patients with metastatic CRC has markedly improved due to patients undergoing advanced surgical resection of localized metastases and advanced systemic chemotherapy $(3,4)$. Leucovorin (FOL) and fluorouracil $(5-\mathrm{FU})$ plus oxaliplatin (1-OHP; FOLFOX), or FOL and 5-FU plus irinotecan (SN-38; FOLFIRI), administered in combination with molecularly-targeted drugs, are used as first-line chemotherapy regimens in the treatment of patients with advanced CRC worldwide $(5,6)$. In recent years, various studies have revealed that the median survival time (MST) of patients with advanced CRC undergoing chemotherapy is $>30$ months when patients are simultaneously administered a combination of numerous cytotoxic agents and molecularly-targeted therapies (7-10).

In previous years, the use of primary tumor location in CRC as a predictive factor for response to therapy has attracted attention. Numerous studies have demonstrated the predictive impact of primary tumor location in CRC (11-13). In embryonic development, the right colon (the cecum, the ascending colon and the 
transverse colon) and the left colon (the descending colon and the sigmoid colon) originate from the midgut and the hindgut, respectively (14). Improved clinical outcomes for patients with left-sided colon cancer (CC) compared with patients with right-sided $\mathrm{CC}$ have been previously reported (11-14). Improved outcomes for patients with left-sided CC are dependent upon molecular tumor biology, particularly when molecularly-targeted agent regimens for palliative chemotherapies are used (15-22). Therefore, in aforementioned studies, the administered chemotherapy regimens always included molecularly-targeted agents. To the best of our knowledge, the impact of primary tumor location as a predictive factor in cytotoxic anticancer agent alone (administration of FOLFOX/FOLFIRI in the absence of molecularly-targeted agents) remains to be determined.

The aim of the present study was to clarify the impact of primary tumor location as a predictive factor for the efficacy of cytotoxic anticancer agents when administered in the absence of molecular target agents using collagen gel droplet-embedded drug sensitivity test (CD-DST).

\section{Materials and methods}

Patients. Between March 2008 and April 2017, tumor specimens were obtained from 133 patients with CRC. Lymph node metastasis with and without distant metastasis was reported in these patients. All patients included in the present study had not received preoperative chemotherapy or chemoradiotherapy prior to enrollment. Written, informed consent for the determination of individual chemosensitivity was obtained from all patients. Approval for the present study was granted by the Tobu Chiiki Hospital Institutional Review Board (Tokyo, Japan; grant no. 02.03.29. \#1).

Methods. The concept of the CD-DST method, it is in vitro assay, is to reproduce the minimum in vivo tumor environment and predict the effect of anticancer drugs on the original primary tumor in vivo. This method was approved by the Japanese Ministry of Health, Labor and Welfare as Practical Diagnostic Assay in 2008 as an assay reimbursed with public medical insurance after assessing the validity, safety and reliability of the assay. CD-DST was performed using a Human Cancer Primary Culture System kit; Primastarä (Kurabo Industries, Ltd., Osaka, Japan). All tumor tissues were excised from primary surgical specimens and subjected to CD-DST. CD-DST analysis was performed to investigate the drug sensitivity of isolated tumor cells cultured in a three-dimensional manner in a small collagen gel droplet, and to determine the sensitivity of the tumors to 5-FU, which was performed in accordance with a protocol previously published by Kobayashi et al $(23,24)$. Each specimen was washed five times with $50 \mathrm{ml}$ saline, which was followed by with an additional five washes with $50 \mathrm{ml}$ antibiotic fluid containing $1.0 \mathrm{mg} / \mathrm{ml}$ piperacillin and $0.5 \mathrm{mg} / \mathrm{ml} \mathrm{kanamycin}$. The transport centrifuge tube was filled with $30 \mathrm{ml}$ of the culture medium containing $1.0 \mathrm{mg} / \mathrm{ml}$ piperacillin, $0.5 \mathrm{mg} / \mathrm{ml}$ kanamycin and $2.5 \mu \mathrm{g} / \mathrm{ml}$ amphotericin B. Tissue samples $(1 \mathrm{~g})$ were incubated for $2 \mathrm{~h}$ at $37^{\circ} \mathrm{C}$ with a cocktail containing $1.0 \%$ dispersion enzyme EZ ${ }^{\mathrm{TM}}$ (Kurabo Industries, Ltd.). Dispersed cell suspensions were inoculated in pre-culture media in collagen-coated flasks (CG-flusk ${ }^{\mathrm{TM}}$; Kurabo Industries, Ltd.) overnight. Surviving tumor cells were subsequently recovered via treatment with $0.05 \%$ collagenase and then embedded in $30 \mu 1$ collagen gel droplets.

Embedded cells were then incubated for $24 \mathrm{~h}$ at $37^{\circ} \mathrm{C}$ in culture media containing either 5-FU $(6.0 \mu \mathrm{g} / \mathrm{ml})$ and 1-OHP $(3.0 \mu \mathrm{g} / \mathrm{ml}$; FOLFOX regimen), or 5-FU $(6.0 \mu \mathrm{g} / \mathrm{ml})$ and $\mathrm{SN}-38$ $(0.2 \mu \mathrm{g} / \mathrm{ml}$; FOLFIRI regimen). Following the removal of the anticancer agent-containing media, cells were additionally cultured for 7 days in serum-free culture media (PCM-2 ${ }^{\mathrm{TM}}$; Kurabo Industries, Ltd.) to prevent the growth of fibroblasts. Surviving cells were stained with neutral red solution and counted using the imaging colorimetric quantification method (Primage $^{\mathrm{TM}}$; Kurabo Industries, Ltd.). The viable cell number ratio between the drug-treated group and the control group, which received no drug treatment, was calculated. A growth rate of $<0.8$ was considered to indicate a successful culture.

Collecting cancer cells. A viable region was taken by a skilled surgeon while avoiding the necrotic area from the excised tumor tissue. It was confirmed by a pathologist that the excised tumor was definitely a cancer tissue. Then, the viable cancer cells were collected through the means of digestion by enzyme and pre-culture method. Cell lines from collected cancer cells were not established.

Validity of the assay. For the control group, when the required minimum number of cells (measured value of the Image Analysis System; 0.1 or more) was present and the relative proliferation ratio in the assay period was 0.8 or more, it was regarded as being performed correctly.

Treatment. Cetuximab or panitumumab was administered prior to cytotoxic chemotherapies: $400 \mathrm{mg} / \mathrm{m}^{2}$ of cetuximab was infused intravenously over $2 \mathrm{~h}$ on day 1 , then $250 \mathrm{mg} / \mathrm{m}^{2}$ over $1 \mathrm{~h}$ every week, and $6 \mathrm{mg} / \mathrm{kg}$ of panitumumab was infused intravenously over 1 h every 2 weeks. Bevacizumab was administered prior to cytotoxic chemotherapies: $5 \mathrm{mg} / \mathrm{kg}$ of bevacizumab was infused over 90 min every 2 weeks. Assuming no adverse reactions, subsequent infusions were administered over a half-hour to $1 \mathrm{~h}$ every other week.

Both histograms and associations between growth inhibition rate (IR) values for each condition were investigated. Cancers proximal or distal of the splenic flexure were designated as right-side or left-side CRC, respectively. The association between the side of the tumor and IR values was determined. The prognosis for patients with right CC vs. patients with left CC was also investigated using patients undergoing palliative chemotherapy who additionally received molecularly-targeted treatment.

Statistical analysis. Histograms were analyzed using the D'Agostino-Pearson omnibus normality test. Data are presented as the median, mean, standard deviation (SD) and standard error (SE) of the mean of IR values. The association between each condition was determined via linear regression analysis. The associations between IR values of patients with right-side tumors and left-side tumors were investigated using the Student's t-test. MST values were calculated using the Kaplan Meier method. Overall survival curve groups were compared using the log rank test. Data are presented as the mean \pm standard deviation and were analyzed using GraphPad Prism software (version 5.04; GraphPad Software, 
Table I. Patient characteristics.

\begin{tabular}{|c|c|c|c|c|c|c|c|}
\hline Primary tumor location & Cecum & $\begin{array}{l}\text { Ascending } \\
\text { colon }\end{array}$ & $\begin{array}{l}\text { Transverse } \\
\text { colon }\end{array}$ & $\begin{array}{l}\text { Descending } \\
\text { colon }\end{array}$ & $\begin{array}{l}\text { Sigmoid } \\
\text { colon }\end{array}$ & Rectum & Total \\
\hline Age, years, mean (range) & $\begin{array}{c}63.1 \\
(42-79)\end{array}$ & $\begin{array}{c}66.9 \\
(46-81)\end{array}$ & $\begin{array}{c}69.0 \\
(36-84)\end{array}$ & $\begin{array}{c}68.1 \\
(55-82)\end{array}$ & $\begin{array}{c}66.5 \\
(47-82)\end{array}$ & $\begin{array}{c}66.0 \\
(37-79)\end{array}$ & $\begin{array}{c}65.5 \\
(36-84)\end{array}$ \\
\hline Sex, male/female & $2 / 5$ & $15 / 8$ & $2 / 5$ & $5 / 5$ & $32 / 8$ & $28 / 18$ & $84 / 49$ \\
\hline \multicolumn{8}{|l|}{ Histological type } \\
\hline Papillary adenocarcinoma & & & & & 1 & 1 & 2 \\
\hline $\begin{array}{l}\text { Well differentiated } \\
\text { adenocarcinoma }\end{array}$ & 3 & 5 & 2 & 2 & 6 & 8 & 26 \\
\hline $\begin{array}{l}\text { Moderately differentiated } \\
\text { adenocarcinoma }\end{array}$ & 4 & 14 & 3 & 6 & 28 & 33 & 88 \\
\hline $\begin{array}{l}\text { Poorly differentiated } \\
\text { adenocarcinoma }\end{array}$ & & & 1 & & 2 & 2 & 5 \\
\hline Mucinous adenocarcinoma & & 4 & 1 & 2 & 3 & 1 & 11 \\
\hline Squamous cell carcinoma & & & & & & 1 & 1 \\
\hline Total & 7 & 23 & 7 & 10 & 40 & 46 & 133 \\
\hline
\end{tabular}

Table II. Palliative chemotherapy patient characteristics.

\begin{tabular}{|c|c|c|c|c|c|c|c|}
\hline Primary tumor location & Cecum & $\begin{array}{l}\text { Ascending } \\
\text { colon }\end{array}$ & $\begin{array}{l}\text { Transverse } \\
\text { colon }\end{array}$ & $\begin{array}{l}\text { Descending } \\
\text { colon }\end{array}$ & $\begin{array}{l}\text { Sigmoid } \\
\text { colon }\end{array}$ & Rectum & Total \\
\hline Age, years, mean (range) & 54 & $\begin{array}{c}63.5 \\
(52-78)\end{array}$ & $\begin{array}{c}74.5 \\
(70-79)\end{array}$ & $\begin{array}{c}68.4 \\
(55-80)\end{array}$ & $\begin{array}{c}64.6 \\
(51-80)\end{array}$ & $\begin{array}{c}64.7 \\
(51-78)\end{array}$ & $\begin{array}{c}65.1 \\
(51-80)\end{array}$ \\
\hline Sex, male/female & $0 / 1$ & $5 / 1$ & $0 / 2$ & $2 / 3$ & $10 / 3$ & $12 / 3$ & $29 / 13$ \\
\hline \multicolumn{8}{|l|}{ Histological type } \\
\hline Papillary adenocarcinoma & & & & & 1 & 1 & 2 \\
\hline $\begin{array}{l}\text { Well differentiated } \\
\text { adenocarcinoma }\end{array}$ & & 1 & & 1 & 1 & 1 & 4 \\
\hline $\begin{array}{l}\text { Moderately differentiated } \\
\text { adenocarcinoma }\end{array}$ & 1 & 5 & 1 & 3 & 9 & 11 & 30 \\
\hline $\begin{array}{l}\text { Poorly differentiated } \\
\text { adenocarcinoma }\end{array}$ & & & 1 & & 1 & & 2 \\
\hline Mucinous adenocarcinoma & & & & 1 & 1 & 1 & 3 \\
\hline Squamous cell carcinoma & & & & & & 1 & 1 \\
\hline Total & 1 & 6 & 2 & 5 & 13 & 15 & 42 \\
\hline
\end{tabular}

Inc., La Jolla, CA, USA). $\mathrm{P}<0.05$ was considered to indicate a statistically significant difference.

\section{Results}

Patients. Patient characteristics are presented in Table I. A total of 42 of the 133 patients received palliative chemotherapy following surgery, the characteristics of whom are presented in Table II. Of the 42 patients, $25 \mathrm{CC}$ patients [right $\mathrm{CC}(\mathrm{n}=7)$ and left $\mathrm{CC}(\mathrm{n}=18)]$ received palliative chemotherapy with molecularly-targeted agents. The number of patients with right $\mathrm{CC}$ who received bevacizumab (Genentech, Inc., South San Francisco, CA, USA) and both bevacizumab and cetuximab (Merck KGaA, Darmstadt, Germany) were five and two, respectively. Of the patients with left $\mathrm{CC}$, nine patients received bevacizumab, two patients received cetuximab, one patient received panitumumab (Amgen, Inc., Thousand Oaks, CA, USA), five patients received both bevacizumab and cetuximab and one patient received both bevacizumab and panitumumab.

Histograms of the individual growth IR values. Histograms of the individual growth IRs (\%) under the conditions of the FOLFOX and FOLFIRI regimens are presented in Figs. 1 and 2, respectively. The median, mean, SD and SE of the mean in the FOLFOX regimen were 58.7, 58.5, 16.7 and 1.45 , respectively. The median, mean, and SD and SE of the mean in the FOLFIRI regimen were 69.1, 66.2, 17.1 and 1.48, respectively. The histograms passed the normality test $(\alpha=0.05$; 


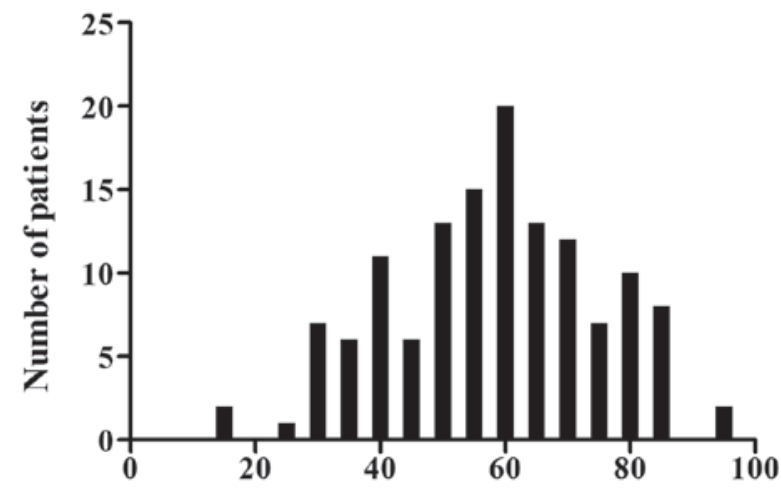

Grow th inhibition rate of FOLFOX (\%)

Figure 1. Histogram of individual growth inhibition rates (\%) was investigated in culture media containing 5-fluorouracil and 1-oxaliplatin at 6.0 and $3.0 \mu \mathrm{g} / \mathrm{ml}$ that was incubated for $24 \mathrm{~h}$ at $37^{\circ} \mathrm{C}$. FOLFOX, leucovorin and fluorouracil plus oxaliplatin.

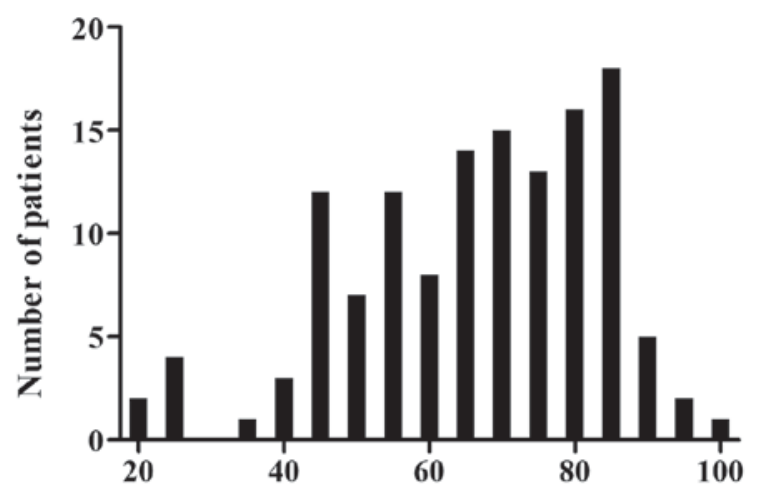

Growth inhibition rate of FOLFIRI (\%)

Figure 2. Histogram of individual growth inhibition rates (\%) was investigated in culture media containing 5-fluorouracil and irinotecan at 6.0 and $0.2 \mu \mathrm{g} / \mathrm{ml}$ that was incubated for $24 \mathrm{~h}$ at $37^{\circ} \mathrm{C}$. FOLFIRI, leucovorin and fluorouracil plus irinotecan.

FOLFOX regimen, $\mathrm{P}=0.68$; FOLFIRI regimen, $\mathrm{P}=0.06$ ). There was a strong correlation between the IRs (\%) of the FOLFOX and FOLFIRI regimens (Fig. 3; $y=0.88 x+14.98, \mathrm{R}^{2}=0.74$ ).

IR values of all patients in the FOLFOX regimen. The IRs (\%) of right- and left-sided tumors including the rectum, were $57.4 \pm 2.5$ and $58.5 \pm 1.8$, respectively $(\mathrm{P}=0.72$; Fig. $4 \mathrm{~A})$. The IRs $(\%)$ of right- and left-sided tumors excluding the rectum $(\mathrm{n}=87)$ were $57.4 \pm 2.5$ and $61.6 \pm 2.4$, respectively $(\mathrm{P}=0.23$; Fig. $4 \mathrm{~B})$.

IR values of all patients in the FOLFIRI regimen. The IRs $(\%)$ of right- and left-sided tumors including the rectum, were $67.0 \pm 2.3$ and $65.8 \pm 1.9$, respectively $(\mathrm{P}=0.69$; Fig. $5 \mathrm{~A})$. The IRs $(\%)$ of right- and left-sided tumors excluding the rectum $(\mathrm{n}=87)$ were 67.0 \pm 2.3 and 69.1 \pm 2.4 , respectively $(\mathrm{P}=0.53$; Fig. 5B).

IR values of 42 patients receiving palliative chemotherapy in the FOLFOX regimen. The IRs (\%) of right- and left-sided tumors including the rectum, were $63.8 \pm 5.4$ and $62.9 \pm 2.8$, respectively $(\mathrm{P}=0.88$; Fig. $6 \mathrm{~A})$. The IRs $(\%)$ of right- and left-sided tumors excluding the rectum $(n=27)$ were $63.8 \pm 5.4$ and 67.0 \pm 3.8 , respectively $(\mathrm{P}=0.64$; Fig. $6 \mathrm{~B})$.

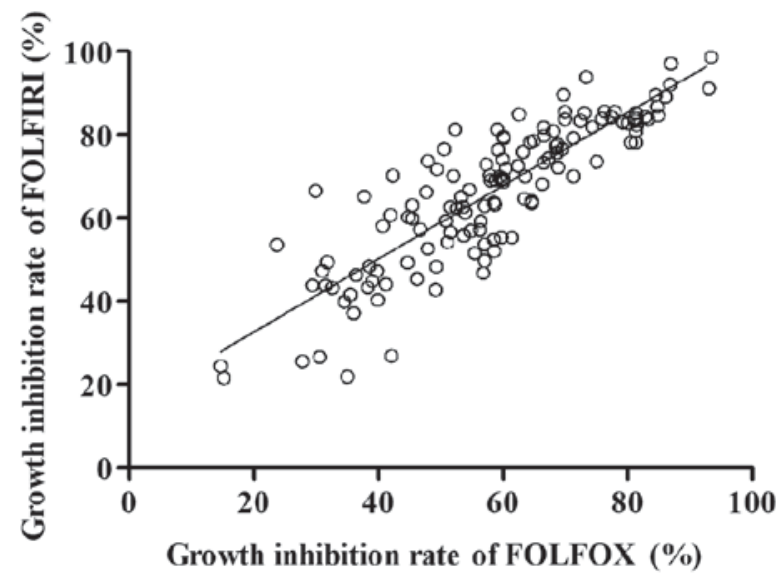

Figure 3. Correlation between individual growth inhibition rates and condition types. FOLFOX, leucovorin and fluorouracil plus oxaliplatin; FOLFIRI, leucovorin and fluorouracil plus irinotecan.

IR values of 42 patients receiving palliative chemotherapy in the FOLFIRI regimen. The IRs (\%) of right- and left-sided tumors including the rectum, were $74.0 \pm 2.6$ and $69.2 \pm 2.8$, respectively ( $\mathrm{P}=0.22$; Fig. $7 \mathrm{~A})$. The IRs (\%) of right- and left-sided tumors excluding the rectum $(n=27)$ were $74.0 \pm 2.6$ and $72.7 \pm 3.5$, respectively $(\mathrm{P}=0.76$; Fig. $7 \mathrm{~B})$.

IR values of 25 patients with $C C$ receiving palliative chemotherapy and treated with molecularly-targeted agents in the FOLFOX regimen. The IRs (\%) of right- and left-sided tumors in the FOLFOX regimen were $61.9 \pm 6.5$ and $67.0 \pm 3.8$, respectively $(\mathrm{P}=0.52$; Fig. $8 \mathrm{~A})$.

IR values of 25 patients with $C C$ receiving palliative chemotherapy and treated with molecularly-targeted agents in the FOLFIRI regimen. The IRs (\%) of right- and left-sided tumors in the FOLFIRI regimen were $74.1 \pm 3.2$ and $72.7 \pm 3.5$, respectively ( $\mathrm{P}=0.77$; Fig. $8 \mathrm{~B})$.

Among all patients, there was no significant difference in the IRs (\%) of the FOLFOX and FOLFIRI regimens using CD-DST between right- and left-sided tumors, including or excluding the rectum.

Prognosis of 25 patients receiving palliative chemotherapy and treated with molecularly-targeted agents. The median follow-up time period of patients suffering from $\mathrm{CC}$ who had been administered palliative chemotherapy and treatment with molecularly-targeted agents $(n=25)$ was 800 days. Furthermore, the MSTs in patients with right CC and patients with left CC were 960 and 1,348 days, respectively (Fig. 9). However, there were no significant differences $(\mathrm{P}=0.11)$.

\section{Discussion}

The CD-DST method is an examination for evaluating the effect of a cross productive anticancer drug on tumors under a culturing condition that minimally reproduces tumor microenvironments in vivo. This reproduction of this microenvironment is to carry out three-dimensional primary culture on a coexistence of cancer cells and fibroblasts derived from tumor tissue with a serum-free medium containing 

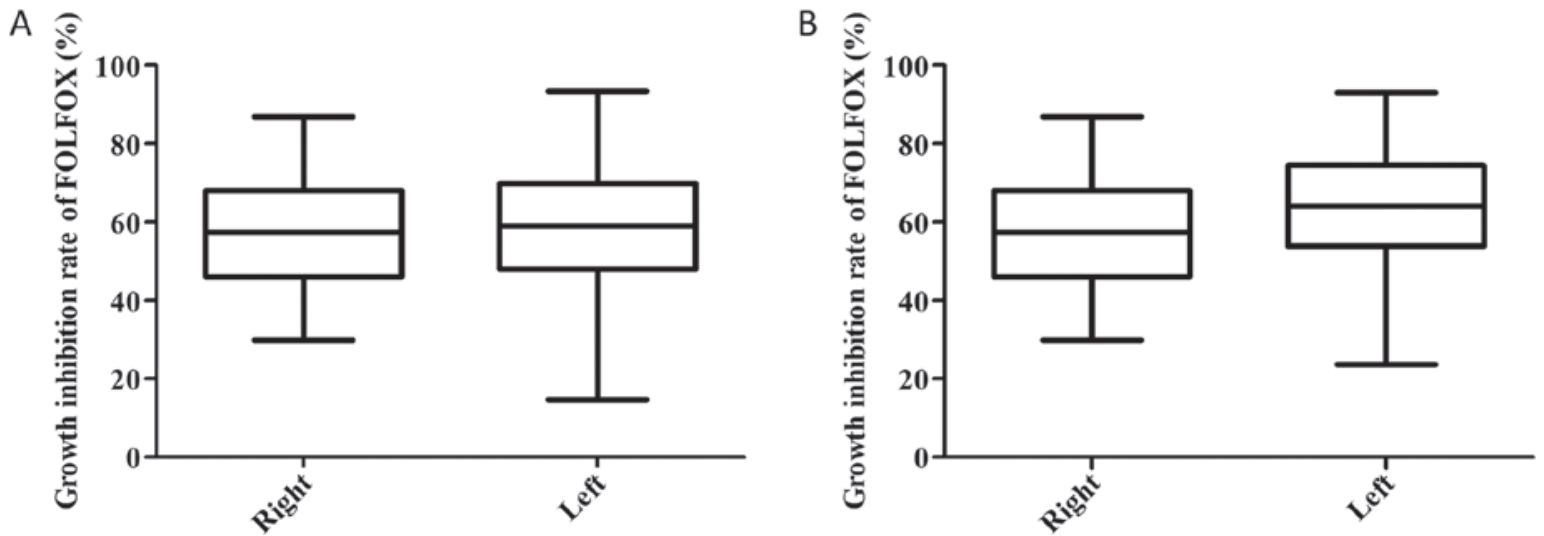

Figure 4. Growth inhibition rates in the FOLOX regimen of right-sided and left-sided tumors in all patients and in 87 patients suffering from colon cancer. In all patients (A) and in 87 patients suffering from colon cancer (B), growth inhibition rates in the FOLFOX regimen (\%) of right-sided and left-sided tumors were investigated in culture media containing 5-fluorouracil and l-oxaliplatin at 6.0 and $3.0 \mu \mathrm{g} / \mathrm{ml}$ that was incubated for $24 \mathrm{~h}$ at $37^{\circ} \mathrm{C}$. FOLFOX, leucovorin and fluorouracil plus oxaliplatin.
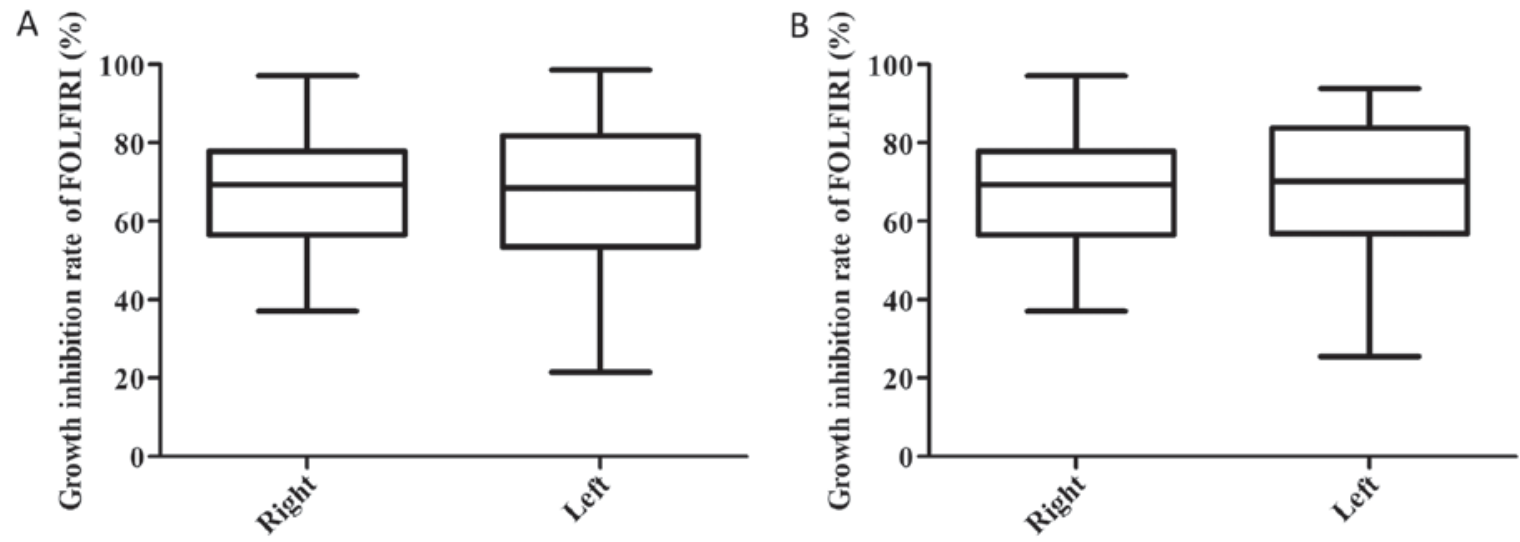

Figure 5. Growth inhibition rates in the FOLFIRI regimen of right-sided and left-sided tumors in all patients and in 87 patients suffering from colon cancer. In all patients (A) and in 87 patients suffering from colon cancer (B), growth inhibition rates in the FOLFIRI regimen (\%) of right-sided and left-sided tumors were investigated in culture media containing 5-fluorouracil and irinotecan at 6.0 and $0.2 \mu \mathrm{g} / \mathrm{ml}$ for $24 \mathrm{~h}$ at $37^{\circ} \mathrm{C}$. FOLFIRI, leucovorin and fluorouracil plus irinotecan.
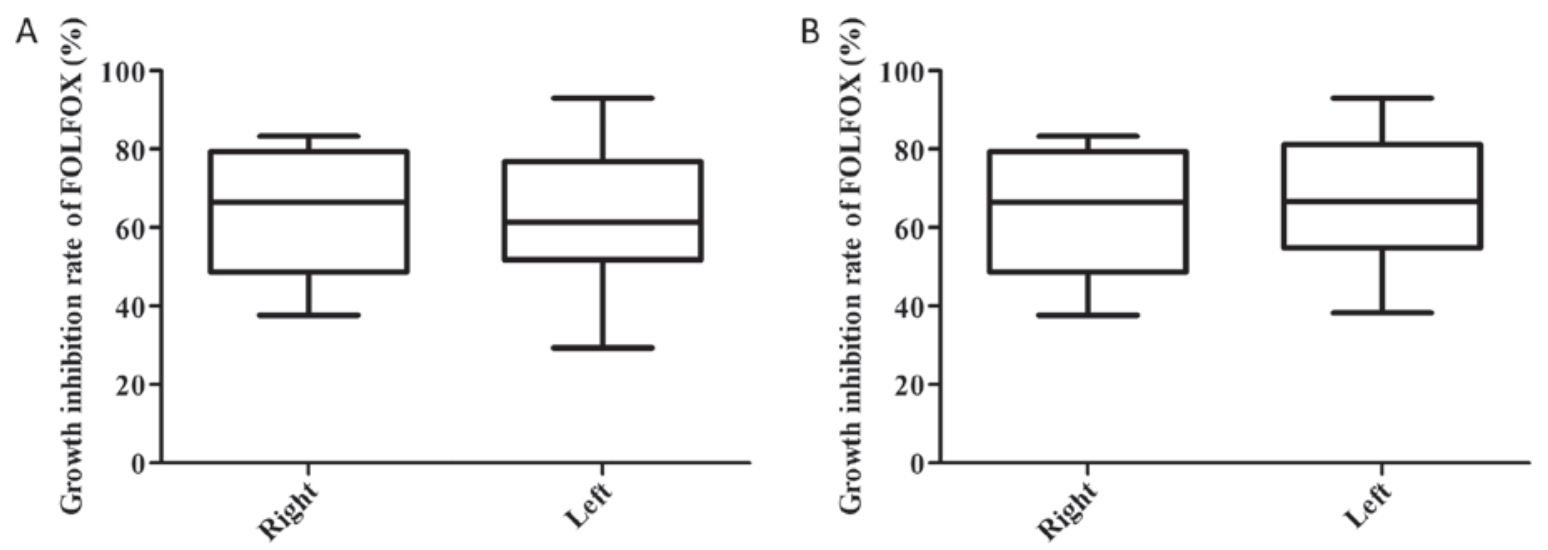

Figure 6. Growth inhibition rates in the FOLOX regimen of right-sided and left-sided tumors in 42 patients with colorectal cancer and in 27 patients with colon cancer. In 42 patients with colorectal cancer receiving palliative chemotherapy (A) and in 27 patients with colon cancer receiving palliative chemotherapy (B), growth inhibition rates in the FOLFOX regimen (\%) of right-sided and left-sided tumors were investigated in culture media containing 5-fluorouracil and 1-oxaliplatin at 6.0 and $3.0 \mu \mathrm{g} / \mathrm{ml}$ that was incubated for $24 \mathrm{~h}$ at $37^{\circ} \mathrm{C}$. FOLFOX, leucovorin and fluorouracil plus oxaliplatin.

various cell growth factors in the collagen gel which is an extracellular matrix $(\mathrm{ECM})$. In order to accurately evaluate chemosensitivity against cancer cells, it is evaluated by 'Image
Analysis System', which utilizes the difference in growth morphology between cancer cells and fibroblasts (25). In the CD-DST method, the primary cancer cells were cultured in 

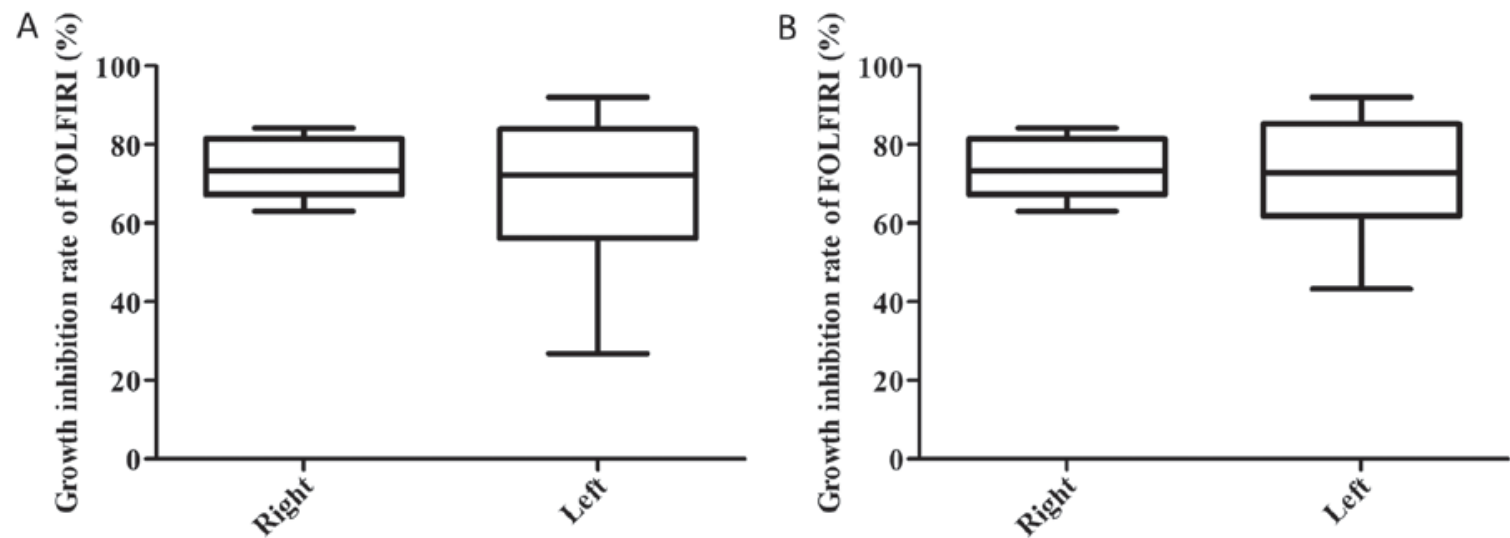

Figure 7. Growth inhibition rates in the FOLFIRI regimen of right-sided and left-sided tumors in 42 patients with colorectal cancer and in 27 patients with colon cancer. In 42 colorectal cancer patients receiving palliative chemotherapy (A) and in 27 colon cancer patients receiving palliative chemotherapy (B), growth inhibition rates in the FOLFRI regimen (\%) of right-sided and left-sided tumors were investigated in culture media containing 5-fluorouracil and irinotecan at 6.0 and $0.2 \mu \mathrm{g} / \mathrm{ml}$ that was incubated for $24 \mathrm{~h}$ at $37^{\circ} \mathrm{C}$. FOLFIRI, leucovorin and fluorouracil plus irinotecan.
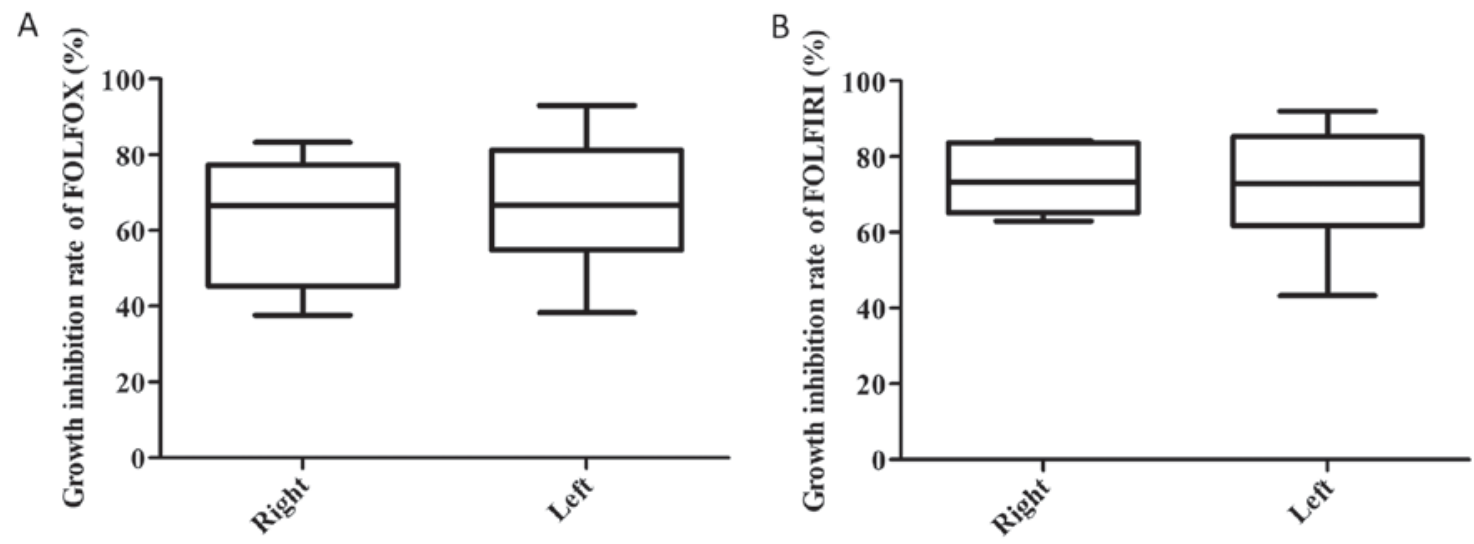

Figure 8. Growth inhibition rates in the FOLOX and FOLFIRI regimens of right-sided and left-sided in 25 patients with colon cancer. (A) In 25 patients with colon cancer receiving palliative chemotherapy and treated with molecularly-targeted agents, growth inhibition rates in the FOLFOX regimen (\%) of right-sided and left-sided tumors were investigated in culture media containing 5 -FU and l-oxaliplatin at 6.0 and $3.0 \mu \mathrm{g} / \mathrm{ml}$ that was incubated for $24 \mathrm{~h}$ at $37^{\circ} \mathrm{C}$. (B) In 25 patients with colon cancer receiving palliative chemotherapy and treatment with molecularly-targeted agents, growth inhibition rates in the FOLFIRI regimen (\%) of right-sided and left-sided tumors were investigated in culture media containing 5-FU and irinotecan at 6.0 and $0.2 \mu \mathrm{g} / \mathrm{ml}$ that was incubated for $24 \mathrm{~h}$ at $37^{\circ} \mathrm{C}$. 5-FU, 5-fluorouracil; FOLFOX, leucovorin and fluorouracil plus oxaliplatin; FOLFIRI, leucovorin and fluorouracil plus irinotecan.

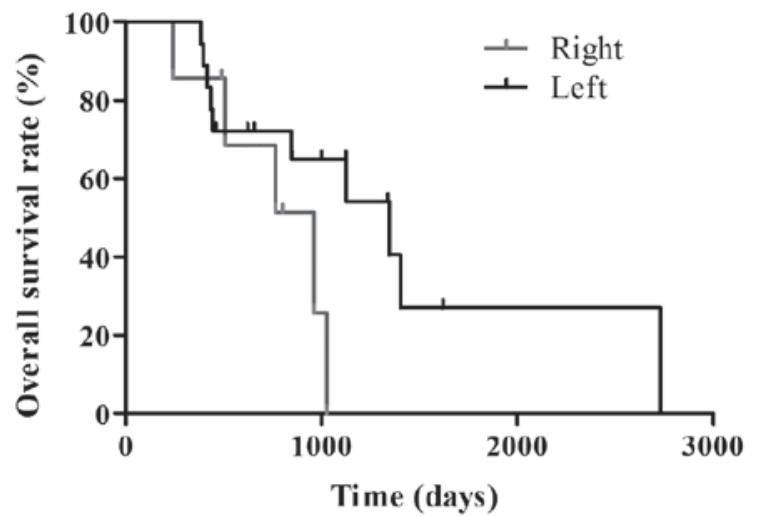

Figure 9. In 25 patients with colon cancer receiving palliative chemotherapy and treated with molecularly-targeted agents, the overall survival rates in patients with left colon cancer (black line) and right colon cancer (gray line) were determined.

serum-free medium. In addition, the serum-free medium does not contain cell growth factors necessary for proliferation of fibroblasts and vascular endothelial cells that may be contaminated. Unlike on collagen gel (2D-culture), fibroblasts have the property of suppressing their proliferation in collagen gel (3D-culture). In the collagen gel, the epithelium-derived cancer cell and the mesenchymal normal cell clearly differ in their proliferation form. The former takes a spherical or thick dendritic form; the latter universally takes a bipolar shape. The fact that spherical colonies are cancer cells has been confirmed by the immunostaining method at the time of development of this assay method (25). Macrophages and lymphocytes, which are typical inflammatory cells, are not adhered on collagen gel coat flasks during preliminary culture within $72 \mathrm{~h}$, so they are always removed by medium change. The cancer cells are thereby purified. As described above, in the CD-DST method, the chemosensitivity of each anticancer agent is evaluated in the primary cell-culture condition of cancer cells which have been collected and purified from fresh tumor tissue.

As mentioned earlier, this assay is intended to 'predict the direct effect of anticancer drugs on tumor tissue', so it is not possible to evaluate the adverse reactions caused by 
chemotherapy. Many side effects caused by administration of anticancer drugs are caused by disorders such as bone marrow, peripheral nerve, gastrointestinal tract, not tumor part. Therefore, the influence of anticancer agents on normal cells on the premise of side effect prediction deviates from our objective. Regarding the influence on normal cells in the tumor tissue, in the CD-DST method, fibroblasts derived from tumor tissue are mixed in the collagen gel. The proliferation of these cells is suppressed by the culture environment and a serum-free medium, but it does not lead to cell death and its physiological activity is maintained. Under this coexisting condition, we can evaluate the effect of anticancer drugs on cancer. Usually, when chemotherapy is performed, patients are monitored for the occurrence and degree of adverse reactions, symptomatic treatment is administered at any time, and symptoms are also managed (26). As we already reported (27-29), the prognosis of the high-sensitive group is good, so the prediction of adverse reactions is not so important, but it seems to be most important to extract the group that can be expected to be effective.

FOLFOX or FOLFIRI in combination with molecularly-targeted drugs represent first-line chemotherapy regimens used for the treatment of advanced CRC globally $(3,4)$. It has been reported that the clinical response rates to FOLFOX and FOLFIRI are equivalent ( $50 \%)(30-33)$. In the present study, there were a number of small differences between the IRs (\%) of the FOLFOX and FOLFIRI regimens in numerous patients. However, there was a strong overall correlation between the IRs (\%) of the two regimen exhibited by the majority of patients $\left(\mathrm{R}^{2}=0.74\right)$. Therefore, this result might show that the efficacies of FOLFOX and FOLFIRI are approximately equivalent in individual. This result also supports the findings of previous studies (30-33).

Improved clinical outcomes for patients with left-sided $\mathrm{CC}$ compared with patients with right-sided $\mathrm{CC}$ have been reported worldwide (11-13). It has been previously demonstrated that improved outcomes for patients with left-sided CC is dependent upon molecular tumor biology, particularly when molecularly-targeted agent regimens are used (15-22). The molecular differences between the right colon and the left colon are notable. Molecularly, right-sided CC and left-sided CC are two different diseases (14). Right-sided CC is associated with defective mismatch repair (MMR) genes, as well as mutations in K-Ras, B-Raf and microRNA-31, whereas left-sided CC is associated with p53, chromosomal instability and expression of N-Ras, microRNA-146a, microRNA-147b and microRNA-1288 (34-36). In clinical practice, molecularly-targeted agents corresponding to identified molecular tumor biology are improving prognostic prediction (15-22). Therefore, primary tumor location may be considered to represent a predictive factor in molecularly-targeted agents. Molecularly-targeted agents should be administered according to molecular tumor biology. However, to the best of our knowledge, there is only one study that has previously investigated whether primary tumor location alone is a predictive factor for the correct use of cytotoxic anticancer agents (15). Boisen et al reported that patients with left-sided CC treated with capecitabin and oxaliplatin (CAPEOX) with bevacizumab exhibited improved clinical outcomes compared with patients suffering from right-sided CC. However, no correlation between primary tumor location and outcome was observed in patients treated with CAPEOX without bevacizumab (15). In recently performed chemotherapy trials, molecularly-targeted agent regimens were revealed to be necessary, particularly in patients receiving palliative chemotherapy $(5,6)$. Therefore, at present, it is difficult to investigate primary tumor location as a predictive factor in patients treated with cytotoxic anticancer agents alone via prospective randomized studies, particularly in patients undergoing palliative chemotherapy. Previously, we have reported the effectiveness of CD-DST for the individualization of first-line treatment in CRC. Therefore, the present study aimed to determine the importance of primary tumor location as a predictive factor in cytotoxic anticancer agent treatment using CD-DST (27-29).

In the present study, there were no significant differences in the IRs (\%) of the FOLFOX and FOLFIRI regimens using CD-DST between right- and left-sided tumors, including or excluding the rectum. These results were consistent with Boisen's study (15). Cytotoxic anticancer agents inhibit the cellular division of cancer cells as well as normal cells (26). 5-FU is an S-phase-specific drug and is active only during specific stages of the cell cycle. The target enzyme of 5-FU is thymidylate synthase, which is the main enzyme associated with DNA synthesis. Therefore, 5-FU inhibits DNA synthesis, which results in RNA dysfunction $(27,37,38)$. Irinotecan is also an S-phase-specific drug that is active only during specific stages of the cell cycle. Irinotecan is metabolized by carboxylesterases to its active metabolite, 7-ethyl-10-hydroxycamptothecin (SN-38). SN-38 functions as a classic topoisomerase I inhibitor by stabilizing the topoisomerase I/DNA cleavable complex, which subsequently blocks DNA replication and results in DNA strand breakages (39). Furthermore, oxaliplatin is a cell-cycle non-specific antineoplastic agent (40). Oxaliplatin is classified as a third-generation platinum compound, following cisplatin and carboplatin. Similar to other platinum compounds, oxaliplatin, cisplatin and carboplatin form crosslinks with DNA strands within cancer cells and inhibit DNA replication and transcription (41). It has been well established that cancer cells attenuate this effect by removing cisplatin from DNA via an MMR mechanism (42). However, it has been previously revealed that oxaliplatin, which is larger in structure than cisplatin, is not removed by MMR and it is difficult to acquire a tolerance to (43-45). It can be suggested that molecular tumor biology may not significantly affect the efficacy of cytotoxic anticancer agents during the cell division stage of cancer cells. Therefore, in patients receiving adjuvant chemotherapy in the absence of treatment with molecularly-targeted agents, improved outcomes for patients suffering from left-sided CC compared with right-sided CC are not observed (46-48). However, of the 25 patients that received palliative chemotherapy as well as treatment with molecularly-targeted agents in the present study, the MSTs in patients suffering from right-sided CC and left-sided CC were 960 and 1,348 days, respectively. This result supports the hypothesis that when administered molecularly-targeted agent regimens, patients suffering from left-sided CC exhibit improved clinical outcomes compared with patients suffering from right-sided $\mathrm{CC}$, depending upon molecular tumor biology, as Boisen et al reported (15). In the CD-DST, there were no significant differences in the IRs (\%) of cytotoxic anticancer agents without molecularly-targeted agents between right- and left-sided colon cancer tumors. Conversely, clinical 
outcomes of left-sided colorectal cancer patients treated with cytotoxic agents and molecularly-targeted agents were better than those of right-sided colon cancer patients. Thus, impact on the prognosis may be due to the effect of molecular target agents. Therefore, primary tumor location may represent a predictive factor for the efficacy of molecularly-targeted agents, rather than a prognostic factor, in patients with CRC.

However, there were several limitations in the present study. Firstly, the sample size was small. However, the proportion of rectal cancers in left-sided colorectal cancers was not small, which might have influenced statistical analysis. Therefore, we had to evaluate the prognosis of only colon cancer patients in palliative chemotherapy with molecularly-targeted agents. A larger sample size would have improved the quality of the data. Secondly, observation periods were short for overall survival rates. The small sample size and the short observation periods may have affected the validity of statistical analyses. Thirdly, we did not evaluate the effects of cytotoxic anticancer agents on normal cell division. Investigation of these effects would have improved the quality of the data.

In conclusion, primary tumor location was revealed to not represent a predictive factor in cytotoxic anticancer agent regimens for patients with $\mathrm{CRC}$. However, improved clinical outcomes for patients with left-sided CC compared with right-sided CC were demonstrated when patients were administered molecularly-targeted agent regimens. Therefore, the results of the present study suggested that molecularly-targeted agents rather than cytotoxic anticancer agents may result in improved clinical outcomes for patients with $\mathrm{CC}$ suffering from left-sided tumors.

\section{Acknowledgements}

Not applicable.

\section{Funding}

No funding was received.

\section{Availability of data and materials}

The datasets used and/or analyzed during the current study are available from the corresponding author on reasonable request.

\section{Authors' contributions}

TO and IN contributed to the conception and design of the present study. KNi, TW, MK, AN, KNa, NS, TS, KK, YA and TO acquired the patient data. TW, MK, AN, KNa, NS, TS, KK, YA and $\mathrm{TO}$ performed the preparation of viable regions from excised tumor tissues. TO analyzed and interpreted the data. KNi and IN were major contributors in writing the manuscript. TO wrote the manuscript. All authors read and approved the final manuscript.

\section{Ethics approval and consent to participate}

Approval for the present study was obtained from the Tobu Chiiki Hospital Institutional Review Board (approval no. 02.03.29. \#1; Tokyo, Japan). Written informed consent for this study was obtained from all patients.

\section{Patient consent for publication}

The patients provided written informed consent for the publication of any associated data.

\section{Competing interests}

The authors declare that they have no competing interests.

\section{References}

1. Ferlay J, Soerjomataram I, Dikshit R, Eser S, Mathers C, Rebelo M, Parkin DM, Forman D and Bray F: Cancer incidence and mortality worldwide: Sources, methods and major patterns in GLOBOCAN 2012. Int J Cancer 136: E359-E386, 2015.

2. Siegel RL, Miller KD, Fedewa SA, Ahnen DJ, Meester RG, Barzi A and Jemal A: Colorectal cancer statistics, 2017. CA Cancer J Clin 67: 177-193, 2017.

3. Adam R, De Gramont A, Figueras J, Guthrie A, Kokudo N, Kunstlinger F,LoyerE, Poston G, Rougier P, Rubbia-BrandtL, et al: The oncosurgery approach to managing liver metastases from colorectal cancer: A multidisciplinary international consensus. Oncologist 17: 1225 1239, 2012.

4. Jones RP, Stättner S, Sutton P, Dunne DF, McWhirter D, Fenwick SW, Malik HZ and Poston GJ: Controversies in the oncosurgical management of liver limited stage IV colorectal cancer. Surg Oncol 23: 53-60, 2014.

5. NCCN: NCCN Clinical Practice Guidelines in Oncology. Colon Cancer. Version 2. 2017. http://www.nccn.org. Accessed February 24, 2018.

6. Yoshino T, Arnold D, Taniguchi H, Pentheroudakis G, Yamazaki K, Xu RH, Kim TW, Ismail F, Tan IB, Yeh KH, et al: Pan-Asian adapted ESMO consensus guidelines for the management of patients with metastatic colorectal cancer: A JSMO-ESMO initiative endorsed by CSCO, KACO, MOS, SSO and TOS. Ann Oncol 29: 44-70, 2018.

7. Fakih MG: Metastatic colorectal cancer: Current state and future directions. J Clin Oncol 33: 1809-1824, 2015.

8. Venook A, Niedzwiecki D, Lenz H, Mahoney M, Innocenti F, O'Neil B, Shaw J, Polite B, Hochster H, Goldberg R, et al: CALGB/SWOG 80405: Analysis of patients undergoing surgery as part of treatment strategy. Ann Oncol 25 (Suppl 4): S1-S41, 2014.

9. Recondo G Jr, Díaz-Cantón E, de la Vega M, Greco M, Recondo G Sr and Valsecchi ME: Advances and new perspectives in the treatment of metastatic colon cancer. World J Gastrointest Oncol 6: 211-224, 2014.

10. Yamada Y, Denda T, Gamoh M, Iwanaga I, Yuki S, Shimodaira H, Nakamura M, Yamaguchi T, Ohori H, Kobayashi K, et al: S-1 and irinotecan plus bevacizumab versus mFOLFOX6 or CapeOX plus bevacizumab as first-line treatment in patients with metastatic colorectal cancer (TRICOLORE): A randomized, open-label, phase III, noninferiority trial. Ann Oncol 29: 624-631, 2018.

11. Wolmark N, Wieand HS, Rockette HE, Fisher B, Glass A, Lawrence W, Lerner H, Cruz AB, Volk H, Shibata H, et al: The prognostic significance of tumor location and bowel obstruction in Dukes B and C colorectal cancer. Findings from the NSABP clinical trials. Ann Surg 198: 743-752, 1983.

12. Loupakis F, Yang D, Yau L, Feng S, Cremolini C, Zhang W, Maus MK, Antoniotti C, Langer C, Scherer SJ, et al: Primary tumor location as a prognostic factor in metastatic colorectal cancer. J Natl Cancer Inst 107: pii: dju427, 2015.

13. Petrelli F, Tomasello G, Borgonovo K, Ghidini M, Turati L, Dallera P, Passalacqua R, Sgroi G and Barni S: Prognostic survival associated with left-sided vs right-sided colon cancer: A systematic review and meta-analysis. JAMA Oncol: Oct 27, 2016 (Epub ahead of print).

14. BoeckxN,JanssensK,VanCampG,RasschaertM,Papadimitriou K, Peeters $\mathrm{M}$ and Op de Beeck K: The predictive value of primary tumor location in patients with metastatic colorectal cancer: A systematic review. Crit Rev Oncol Hematol 121: 1-10, 2018.

15. Boisen MK, Johansen JS, Dehlendorff C, Larsen JS, Osterlind K, Hansen J, Nielsen SE, Pfeiffer P, Tarpgaard LS, Holländer NH, et al: Primary tumor location and bevacizumab effectiveness in patients with metastatic colorectal cancer. Ann Oncol 24: 2554-2559, 2013 
16. Brulé SY, Jonker DJ, Karapetis CS, O'Callaghan CJ, Moore MJ, Wong R, Tebbutt NC, Underhill C, Yip D, Zalcberg JR, et al: Location of colon cancer (right-sided versus left-sided) as a prognostic factor and a predictor of benefit from cetuximab in NCIC CO.17. Eur J Cancer 51: 1405-1414, 2015.

17. Shen H, Yang J, Huang Q, Jiang MJ, Tan YN, Fu JF, Zhu LZ, Fang XF and Yuan Y: Different treatment strategies and molecular features between right-sided and left-sided colon cancers. World J Gastroenterol 21: 6470-6478, 2015.

18. Holch JW, Ricard I, Stintzing S, Modest DP and Heinemann V: The relevance of primary tumour location in patients with metastatic colorectal cancer: A meta-analysis of first-line clinical trials. Eur J Cancer 70: 87-98, 2017.

19. Tejpar S, Stintzing S, Ciardiello F, Tabernero J, Van Cutsem E, Beier F, Esser R, Lenz HJ and Heinemann V: Prognostic and predictive relevance of primary tumor location in patients with RAS wild-type metastatic colorectal cancer: Retrospective analyses of the CRYSTAL and FIRE-3 trials. JAMA Oncol: Oct10, 2016 (Epub ahead of print).

20. Aljehani MA, Morgan JW, Guthrie LA, Jabo B, Ramadan M, Bahjri K, Lum SS, Selleck M, Reeves ME, Garberoglio C and Senthil M: Association of primary tumor site with mortality in patients receiving bevacizumab and cetuximab for metastatic colorectal cancer. JAMA Surg 153: 60-67, 2018.

21. Ishihara S, Murono K, Sasaki K, Yasuda K, Otani K, Nishikawa T, Tanaka T, Kiyomatsu T, Kawai K, Hata K, et al Impact of primary tumor location on postoperative recurrence and subsequent prognosis in nonmetastatic colon cancers: A multicenter retrospective study using a propensity score analysis. Ann Surg 267: 917-921, 2018.

22. Yamashita S, Brudvik KW, Kopetz SE, Maru D, Clarke CN, Passot G, Conrad C, Chun YS, Aloia TA and Vauthey JN: Embryonic origin of primary colon cancer predicts pathologic response and survival in patients undergoing resection for colon cancer liver metastases. Ann Surg 267: 514-520, 2018

23. Kobayashi H, Tanisaka K, Doi O, Kodama K, Higashiyama M, Nakagawa H, Miyake M, Taki T, Hara S, Yasutomi M, et al: An in vitro chemosensitivity test for solid tumors using collagen gel droplet embedded cultures. Int J Oncol 11: 449-455, 1997.

24. Kobayashi H, Higashiyama M, Minamigawa K, Tanisaka K, Takano T, Yokouchi H, Kodama K and Hata T: Examination of in vitro chemosensitivity test using collagen droplet culture method with colorimetric endpoint quantification. Jpn J Cancer Res 92: 203-210, 2001.

25. Kobayashi H: Development of a new in vitro chemosensitivity test using collagen gel droplet embedded culture and image analysis for clinical usefulness. Recent Results Cancer Res 161: 48-61, 2003.

26. Corrie PG: Cytotoxic chemotherapy: Clinical aspects. Medicine 36: 24-28, 2008.

27. Ochiai T, Nishimura K, Watanabe T, Kitajima M, Hashiguchi T, Nakatani A, Marusasa T, Muraki A, Nagaoka I and Futagawa S: Leucovorin and fluorouracil plus oxaliplatin or leucovorin and fluorouracil plus irinotecan as individualized first-line therapy based on a drug sensitivity test. Exp Ther Med 1: 325-329, 2010

28. Ochiai T, Nishimura K, Watanabe T, Kitajima M, Nakatani A Inou T, Washio M, Sakuyama N, Sato T, Kishine K, et al: Individualized chemotherapy for colorectal cancer based on the collagen gel droplet-embedded drug sensitivity test. Oncol Lett 4: 621-624, 2012.

29. Ochiai T, Nishimura K, Watanabe T, Kitajima M, Nakatani A, Nagayasu K, Naito S, Sato T, Kishine K, Abe Y, et al: Impact of the individualization of the first-line chemotherapy for advanced colorectal cancer based on collagen gel droplet-embedded drug sensitivity test. Oncol Lett 14: 6045-6052, 2017.

30. Tournigand $\mathrm{C}$, André $\mathrm{T}$, Achille E, Lledo G, Flesh M, Mery-Mignard D, Quinaux E, Couteau C, Buyse M, Ganem G, et al: FOLFIRI followed by FOLFOX6 or the reverse sequence in advanced colorectal cancer: A randomized GERCOR study. J Clin Oncol 22: 229-237, 2004.

31. de Gramont A, Figer A, Seymour M, Homerin M, Hmissi A, Cassidy J, Boni C, Cortes-Funes H, Cervantes A, Freyer G, et al: Leucovorin and fluorouracil with or without oxaliplatin as first-line treatment in advanced colorectal cancer. J Clin Oncol 18 2938-2947, 2000.

32. Goldberg RM, Sargent DJ, Morton RF, Fuchs CS Ramanathan RK, Williamson SK, Findlay BP, Pitot HC and Alberts SR: A randomized controlled trial of fluorouracil plus leucovorin, irinotecan, and oxaliplatin combinations in patients with previously untreated metastatic colorectal cancer. J Clin Oncol 22: 23-30, 2004.
33. Douillard JY, Cunningham D, Roth AD, Navarro M, James RD, Karasek P, Jandik P, Iveson T, Carmichael J, Alakl M, et al: Irinotecan combined with fluorouracil compared with fluorouracil alone as first-line treatment for metastatic colorectal cancer: A multicentre randomized trial. Lancet 355: 1041-1047, 2000.

34. Lee MS, McGuffey EJ, Morris JS, Manyam G, Baladandayuthapani V, Wei W, Morris VK, Overman MJ, Maru DM, Jiang ZQ, et al: Association of $\mathrm{CpG}$ island methylator phenotype and EREG/AREG methylation and expression in colorectal cancer. Br J Cancer 114: 1352-1361, 2016.

35. Kim SE, Paik HY, Yoon H, Lee JE, Kim N and Sung MK: Sexand gender-specific disparities in colorectal cancer risk. World $\mathbf{J}$ Gastroenterol 21: 5167-5175, 2015.

36. Sinicrope FA, Mahoney MR, Smyrk TC, Thibodeau SN, Warren RS, Bertagnolli MM, Nelson GD, Goldberg RM, Sargent DJ and Alberts SR: Prognostic impact of deficient DNA mismatch repair in patients with stage III colon cancer from a randomized trial of FOLFOX-based adjuvant chemotherapy. J Clin Oncol 31: 3664-3672, 2013.

37. Ochiai T, Nishimura K, Noguchi H, Kitajima M, Watanabe T, Nagaoka I and Futagawa S: Prognotic impact of orotate phosphoribosyl transferase among 5-fluorouracil metabolic enzymes in resectable colorectal cancers treated by oral 5-fluorouracil-based adjuvant chemotherapy. Int J Cancer 118: 3084-3088, 2006.

38. Ochiai T, Umeki M, Miyake H, Iida T, Okumura M, Ohno K, Sakamoto M, Miyoshi N, Takahashi M, Tsumura H, et al: Impact of 5-fluorouracil metabolizing enzymes on chemotherapy in patients with resectable colorectal cancer. Oncol Rep 32: 887-892, 2014

39. Minderman H, Conroy JM, O'Loughlin KL, McQuaid D, Quinn P, Li S, Pendyala L, Nowak NJ and Baer MR: In vitro and in vivo irinotecan-induced changes in expression profiles of cell cycle and apoptosis-associated genes in acute myeloid leukemia cells. Mol Cancer Ther 4: 885-900, 2005.

40. Lévi F, Metzger G, Massari C and Milano G: Oxaliplatin: Pharmacokinetics and chronopharmacological aspects. Clin Pharmacokinet 38: 1-21, 2000.

41. Fink D, Zheng H, Nebel S, Norris PS, Aebi S, Lin TP, Nehmé A, Christen RD, Haas M, MacLeod CL and Howell SB: In vitro and in vivo resistance to cisplatin in cells that have lost DNA mismatch repair. Cancer Res 57: 1841-1845, 1997.

42. Fink D, Nebel S, Aebi S, Zheng H, Cenm B, Nehmé A, Christen RD and Howell SB: The role of DNA mismatch repair in platinum drug resistance. Cancer Res 56: 4881-4886, 1996.

43. Chaney SG, Campbell SL, Bassett E and Wu Y: Recognition and processing of cisplatin- and oxaliplatin-DNA adducts. Crit Rev Oncol Hematol 53: 3-11, 2005.

44. Kiyonari S, Iimori M, Matsuoka K, Watanabe S, Morikawa-Ichinose T, Miura D, Niimi S, Saeki H, Tokunaga E, Oki E, et al: The 1,2-diaminocyclohexane carrier ligand in oxaliplatin induces p53-dependent transcriptional repression of factors involved in thymidylate biosynthesis. Mol Cancer Ther 14: 2332-2342, 2015.

45. Ramachandran S, Temple B, Alexandrova AN, Chaney SG and Dokholyan NV: Recognition of platinum-DNA adducts by HMGB1a. Biochemistry 51: 7608-7617, 2012.

46. Yoshida M, Ishiguro M, Ikejiri K, Mochizuki I, Nakamoto Y, Kinugasa Y, Takagane A, Endo T, Shinozaki H, Takii Y, et al: S-1 as adjuvant chemotherapy for stage III colon cancer: A randomized phase III study (ACTS-CC trial). Ann Oncol 25: 1743-1749. 2014.

47. André T, de Gramont A, Vernerey D, Chibaudel B, Bonnetain F, Tijeras-Raballand A, Scriva A, Hickish T, Tabernero J, Van Laethem JL, et al: Adjuvant fluorouracil, leucovorin, and oxaliplatin in stage II to III colon cancer: Updated 10-year survival and outcomes according to BRAF mutation and mismatch repair status of the MOSAIC study. J Clin Oncol 33: 4176-4187, 2015.

48. Hamaguchi T, Shimada Y, Mizusawa J, Kinugasa Y, Kanemitsu Y, Ohue M, Fujii S, Takiguchi N, Yatsuoka T, Takii Y, et al: Capecitabine versus S-1 as adjuvant chemotherapy for patients with stage III colorectal cancer (JCOG0910): An open-label, non-inferiority, randomised, phase 3, multicentre trial. Lancet Gastroenterol Hepatol 3: 47-56, 2018. 